\section{An Aùtress}

on

\section{SOME PRACTICAL POINTS IN THE TREAT. MENT AND CLINICAL HISTORY OF}

\section{EARLY SYPHILIS}

Deivered before the Manchester (West) Division, British Medical association.

By WILLIAM COATES, C.B., M.R.C.S.

THF study of early syphilis is fall of interest, but its range is so vast that it is but a fringe of the subject that can be dealt with to-day. No disease presents more difficalt problems for solution, or more complicated symptoms for treatment. These concern not only the family practitioner : every branch of the kingdom of medicine is invaded, and each specialist sees, and must be alive to, its early and remote effecte. It respects no class : rich and poor, old and young, peer and peasant, the depraved and the chaste, are alike counted amongst its victims. Its effects may be so lasting as to influence all periods of a man's life, and they bulk as a great factor in determining the vitality, affluence, prosperity, and happiness of a race.

The Curability of Syphilis.

In the large majority of cases, and judged on lines that apply to other diseases, syphilis is curable, and it is unnecessarily cruel to teach otherwise. Occasionally cases occur that resist treatment; and others, apparently immune for years, suddenly break out in symptom. The syphilitic virus sometimes assails a constivution with an idiosyncrasy favourable to its development and multiplica. tion; then, indeed, it is difficult to cure. But by far the larger number of cases of syphilis, if seen early and treated rightly, remain well to all intents and purposes, though the longer the treatment is delayed the more difficult and protracted is the recovery. Small.pox, typhoid and scarlet fevers, or a broken leg leave conditions which sometimes disclose themselves after a lapse of many years, yet none would regard these as incurable. Why should an occasional late reminder of an early syphilis make us class this disease under a different heading?

If after treatment no retarn of symptoms occurs, and if subsequently the patient marries and brings up a healthy family, a cure surely may be assumed.

Let me recall one out of a number of such cases that have been under my care for many years.

Case illustrating the Curability of Syphilis.

In March, 1884, a business man aged 26 consulted me. He had an indurated sore on the froenum; hard, enlarged, and painless glands in both groins; an inflamed and ulcerated painless glands in both groins; an inflamed and ulcerated throat; and a roseolous eruption on the abdomen, back, and
sides. He took $1 \frac{1}{2}$ grains of grey powder with 1 grain of Dover's sides. He took $1 \frac{1}{2}$ grains of grey powder with I grain of Dover's
powder three times daily continuously for two years. After the first three months of treatment he had no return of symptoms. Three years afterwards he wished to marry. On my advice he renewed his treatment for six months as a precaution. H has now been married twenty years, and has six children-the youngest 8 , the eldest 19 . Neither in himself, his wife, nor children has any sign of syphilis been observed.

Another point bears somewhat on the curability of syphilis, and introduces a further topic of interest. It has long been taught-and this teaching has been confirmed by the experimental inoculation of apes-that a person suffering from symptoms of syphilis is immune from a second attack; that so long as syphilis exists, primary syphilis, followed by secondaries, will not occur.

A few cases have been recorded which show that this is not an absolutely infallible rule, just as occasionally one attack of scarlet fever or measles may be followed by another; but such cases are exceedingly rare, and for practical purposes may be put aside. Personally I have never seen fresh primary or secondary syphilis occar in a patient presenting any symptoms of the disease.

It is not impossible, however, to see a second attack in a patient cured of a former one. This is rare, and other conditions to be alladed to later, such as recurring chancres and gummata, cause mistakes; but I have notes of seven cases, several of which I will relate.
Primary and Secondary Syphilis occurring a Second Time.

1. A man aged 22 consulted me on November 30th, 1898, with an indurated single sore on the left of the corona, bullet glands in the groin and neck, mucous patches on throat and mouth, and a papular eruption on forehead and body. He took 1 grain of grey powder four times daily for three months, and afterwards three times daily until the end of two years. No symptoms were seen after the first year of treatment. On September 15th 1903-that is, five years after his first attack and nearly three years after the cessation of treatment-he appeared with an indurated sore on the right side of the prepuce 1 in. from the corone and hard enlarged glands in the groing corona, and hard enlarged glands in the groins. He had been exposed five weeks before. Though it was apparently an infecting sore, under the circumstances events were awaited. affected, a papulo-squamous eruption developed, he lost a large affected, a papulo-squamous eruption developed, he lost a large quantity of hair, and passed through a much mo

of secondary syphilis than on the first occasion. 2. A man aged 24 came to me on January 10th, 1894, with a a red throat, and a roseolous eruption. He zealously carried out a two years' conrse of continuous treatment with Hutchinson' pills and was cured. On March $15 \mathrm{th}, 1900$, he presented himsel with an indurated sore just above the corona after frequent exposures. In three weeks his throat became ulcerated, papular eruption broke out; later his hands showed palmar psoriasis. This was a troublesome case to treat, but ultimately psoriasis. This was a troublesome case to tre

3. J. D. aged 40, consulted me on May 18th, 1906, for an indurated sore on the prepuce. He was married, and might hace had primary and secondary syphilis in 1888, and taken a two years' course of treatment under a well-known London specialist, local treatment alone was given. In a fortnight, however, a scaly papular eruption appeared all over his body, his tonsils were covered with symmetrical ulceration, and he lost much of his hair. His symptoms ware troublesome, but after a two years' course of grey powder he has remained well.

The last case that I will mention is that of a man who married after his first cure, and did not infect his wife until he contracted a second attack.

4. A man consulted me on May 1st, 1888, with a primary indurated sore just above the corona, to the right, symmetrical ulceration of tonsils, enlarged and hardened glands, and a roseolous eruption. $\mathrm{He}$ took grey powder in small doses for roseolous eruption. He took grey powder in small doses for he left Manchester and neglected treatment. On December he lefter ancerion on 2nd, 1889, he returned to me with a gummatous ulceration on the right leg and rupial spots on his body. He took a mixture containing potassium iodide and mercury perchloride for two years, and was cured. In 1892 he married a lady whose ovaries had both been removed by a gynaecological specialist, otherwise I doubt not that he would have had children, as both remsined well. On August 10th, 1906, he came to me with an indurated sore on the margin of the meatus, with bullet glands ulcers on his tonsils, and a papulo-squamous eruption. He had been exposed nine weeks .before, and had been in contact with his wife. He brought her to me on August 24th, 1906, and she had an indurated sore at the posterior fourchette with bullet glands and a roseolous eruption.

The above four cases illustrate second attacks of syphilis occurring in subjects cured of a previous attack and in each the second primary sore occurred in a different position to the earlier one, and was followed by secondary symptoms.

\section{Relapsing Chancres.}

The existence of an indurated sore in a person who has had syphilis is not, however, in itself alone evidence of a second attack; and this discloses a very interesting clinical phenomenon. Chancres not infrequently relapse, thus indicating, though not always, that treatment is not carried out. These recurrences are always in the site of the original sore. A small gumma in the corona, and an ulceration over a local lymphatic inflammation on the penis, may also resemble a primary sore. But, strange to say, a primary sore may also recur whilst a patient is actually under treatment.

This case, recently under my care, bears on these points :

Chancre Recurring during Treatment.

A man, H. G.. aged 34, came to me on February 3rd, 1909, with a hard sore on the right side of the prepuce just above the corona, and well-marked secondary symptoms. He was treated with intramuscular injections of 1 grain of calomel, and afterwards with an intramnjenlar injection of 1 grain of metallic mercury Lambkin's cream) fortnightly. He was free from symptoms in four months, but treatment was of course continued. In December he called my attention to the seat of the primary sore, which had returned, was as large as a threepenny piece, indurated, and eroded. He denied fresh exposure. Mercury atoxylate (1 grain) was substituted for the mercury as an injection, and small doses of potassium iodide were given by the month. In six weeks the induration had disappeared, and so far it has not returned. 
Relapse after Long Immunity.

There is still another class of case which demands con. sideration when we study our attitude in advising patients who have been apparently cured of syphilis. Unhappily, after an immunity-practically a cure-for many years, a man who has had syphilis, undergone the soundest treatment, married, and begotten healthy children, may develop symptoms. Is this man to be "branded "during all these years as " uncured"? Is he to be deprived of all the blessings of family life? I think not. The longer, 8s a rule, that marriage is delayed the better-the virus becoming more attenuated, perhaps; but it seems as unreasonable to forbid a man who has had rheumatic fever or diphtheria to marry, as to prevent one who has been properly treated for syphilis, and in whom, after a reasonable lapse of time, no syrnptoms have appeared. The following cases will illustrate my meaning.

Immonity from Syphilis for Twenty Years, and then an Outburst. Was Marriage justifiable?

On March 1st, 1886, I was called to a man aged 50 who was suffering excruciating pain on the inside of his left knee. He had felt pain for several days, but it had developed acutely during the night. He had never had rheumatism. The knee felt hot, and there was slight effusion into the joint. The tem. perature was $103^{\circ}$, pulse quick. He was treated for several days with salicylates, and it was necessary to give morphine hypodermically. No improvement occurred, and on March 5th the fact was elicited that he had been treated twenty years previouly for primd viously for primary and secondary syphilis by a specialist. $H$ remembered no symptoms after the first few months. It wa remarkable how the severe pain was almost instantly relieved by a few doses of potassium iodide and mercury; he resumed his work in a week, continued treatment for many months, an remained well. He had been married seventeen years; he had four children whose ages ranged from 7 to 16, and in not one of them nor in his wife could I detect any sign of syphilis.

Surely this man had done no wrong in marriage. It is, however, a good illustration of Professor Fournier's fina advice to his patients, which is, "If ever you are ill, no matter how far from now, nor whatever the disorder may be, tell your doctor plainly that you have had syphilis tell him ten times rather than once."

Immunity from Syphilis for Eighteen Years : Its Relation to Marriage.

A man aged 45 consulted me on April 13th, 1909, sufferin from a painful swelling of the right foot. It had been noticed for three months, but it was increasing rapidly and now had inflamed. He had been under some treatment. The foot was swollen, red, and oedematous, and a large ill-deflned gammatous mass occupied its dorsum. It appeared as if about to break down. He admitted syphilis eighteen years before, and treatment by a London specialist. Under large doses of potestreatment a large doses of potasmass disappear mase over the centra metatarsal bones, which has now almost gone. This gentleman 12 and 14 ; in neither could I detect a sign of syphilis, nor in his 12 and

When called upon to advise regarding the curability of syphilis and on the subject of marriage after an attack, it is well to recall cases such as these.

Early Diagnosis and Treatment.

It is a well-recognized clinical fact that the earlier syphilis is treated the more amenable it is. Symptoms, both in the earlier and later stages, are less severe, and, if not entirely prevented, they may be greatly modified. The later treatment is begun the mere rebellions is the disease, the more frequent are its relapses. The moment the diagnosis is made internal treatment should begin. Every sore is not syphilitic; one is followed in weeks or months by symptoms of constitutional infection-another by none.

In military practice, until the discovery of the Spiro. chaeta pallida and Wassermann's reaction, it was the custom to refrain from the constitutional treatment of uny sore until " secondaries" appeared. In my earlier days it was tanght that there were two distinct and well.defined local sores-one a hard and one a soft chancre, the former always a forerunner of syphilis, the latter never-the clinical features of each being so characteristic that a few simple rules would distinguish them. 'Now the doctrine has changed. It is taught that, although a hard sore is usually followed by syphilis, this is not to be relied upon, and that a soft sore may be-in fact, often is-followed by real syphilis. Rollet first demonstrated that the infection of the soft sore and of syphilis might occur together, a mixed chancre or chancres being the result. During a long connexion with the military hospital at the Hulme Barracks I watched the development of early syphilis in many cases, and although it is impossible clinically to foretell whether a non-indurated sore or sores forerun syphilis, it is possible to be reasonably assured that a certain indurated sore will be followed by constitutional symptoms. When the incubation period coincides, and no local caustic has been applied, and if the glands are hard, painless, and enlarged (one rarely sees a chancre without glandular signs), the diagnosis is clear. A gradually increasing, painless, well-defined induration is practically always followed by syphilis.

It is now generally accepted that the Spirochaeta pallida, discovered by Schaudinn in 1905 , and confirmed later by Hoffman and many others, is the cause of syphilis. This discovery opens out a new field for future investigation, and promises to solve many problems. More then twenty years before this discovery Sir Jonathan Hatchinson rote:

The disease depends upon a living and specific microbe; it is contagious or transmissible only so long as that microbe retains its vitality. . . That this discovery is in reserve for some future investigator $I$ have the utmost confidence in believing.

How true a prophecy, and how gratifying that this great master has lived to see its fulfilment!

Though we must recognize that the spirochaeta pallida is the organism which causes syphilis, and that its demon stration makes immediate internal treatment necessary and that its presence can be discovered in sores which otherwise could not at the same stage be declared con. stitutional, still its discovery, and especially its distinction from other spirochaetes which it resembles, require special skill. Until recently it has been the work of the highly trained microscopist, not always available, to prove its existence. Latterly, however, simpler methods of demonstration - "the dark ground illumination"-have been introduced, which will bring more positive diagnosis within reach of all.

But still, the inability to discover the Spirochaeta pallida in a primary lesion is not conclusive evidence of the absence of syphilis. During the last twelve months I have seen one case of a doubtful chancre, which later was followed by "secondaries," in which a distinguished bacteriologist failed to discover any signs of the Spirochaeta pallida. and two other cases, each with an indurated sore and secondary symptoms, in which tho micro-organism could not be found.

The serum diagnosis of syphilis-the so-called Wassermann's reaction, which, if positive, indicates that the individual is suffering from ayphilis-is not at the present time to be relied upon to determine the nature of a sore nor to guide the practitioner in its treatment. The reaction is invariably absent in non-syphilitic subjects, but it is also absent in 15 per cent. of syphilitic cases at any stage, and in the first six or eight weeks of the disease it is rarely present at all. I myself have seen a well-marked case of syphilis in an active state in which the reaction was negative, even though sought for by an expert; and asr it is during the first few weeks of infection that the practi. tioner needs experimental help in his diagnosis, much advance in this research is necessary before it becomes of much practical value in early stages.

I contend that although we are not justified in pronouncing any sore or sores as nop-syphilitic, we are right in assuming from clinical evidence alone that certain sores will be followed by constitutional symptoms, and in instituting treatment before their development. There are sores which are unquestionably syphilitic; there are others which are doubtful, and in the latter we must. be content to watch and wait, unless the Spirochaeta pallida be found, or Wassermann's reaction be positive.

The Imprudence of Starting Constitutional Treatment before a Positive Diagnosis of Syphilis is made.

Though a diagnosis can be made, and treatment begun in many cases of primary eyphilis, it is equally important to withhold internal treatment in any case of doubt. Hopeless confusion and loss of prestige to the surgeon and injury to the patient follow a contrary course, Jet how often a foolish and half-hearted treatment is adopted. The following are tiro out of many similar cases. 
Case showing that Internal Treatment should not be Employed before Certain Diagnosis is made.

A man, aged 22, consulted me on March 29th, 1909. He had flat grey patches on both tonsils, enlarged submaxillary, subocipital and mastoid glands, and scattered papular spots on his body. The penis showed four reddish-brown scars on the glans, but no induration or sore. He stated that early in the glans, but no induration or sore. He stated that early in the
previous August, noticing several discharging sores on his previous August, noticing several discharging sores on his penis, which had appeared a few days after exposure, he consulted a doctor. He was assured that they were soft soresThere was no improvement in a fortnight, so pills were givendoubtless mercurial. His symptoms disappeared and in six weeks he was pronounced cured. His present condition had been developing for three weeks. This was obviously a case of delayed secondary syphilis. The doctor had been uncertain too hasty in giving mercury, then, doubtful of syphilis, he had yielded the issue to fate.

Case Ilustrating Too Early Conclusion from a Sore-and the Value of Mercury Atoxylate in Rapidly Removing Symptoms.

A more striking case will emphasize this point. A man, A.B., aged 30 , consulted me on January 5 th, 1910 . He was to be married the following day. Two months before he had several sores on his penis. His doctor cured them with a lotion and pronounced marriage to be safe. A rash appeared a week agopronounced marriage to be safe. A rash appeared a week agohis mother suffered. I found he had a coppery squamo-papular his mother suffered. I found he had a coppery squamo-papular ulcers-and enlarged and painless glands. Unfortunately, against all advice he married, with what result it is too early to say. His own eymptoms have disappeared most remarkably under the intramuscular injections of mercury atoxylate.

Complaints are often heard that patients refuse treatment after symptoms have disappeared, but this is exceptional in my own experience. Once win the confi. dence of your patient, convince yourself of the necessity, and a straightforward statement of the need for long treatment and the consequences of neglect will usually bind your patient to you until he is cured.

Treatment of the Local Sore.

The investigations following the discovery of the Spirochaeta pallida will do much to settle one long-fought controversy, namely, whether the excision or destruction of the primary sore has any influence in preventing constitational symptoms. It was formerly the practice of many surgeons to adopt severe local measures - either by cautery, caustic, acid, or the knife-hoping thereby to abort the disease. I have often applied acid nitrate of mercury or liquid carbolic acid to an indurated chancre, but long since abandoned such local measures in any attempt to check the disease. If a chancre has developed to an extent sufficient to declare it to be an infecting one it seems useless to destroy it. It is questionable whether a chancre can be diagnosed as syphilitic before general infection has occurred, which in some cases takes place coincidently with the earliest formation of the sore. Although it is possible that the blood may be direotly inoculated with syphilitic poison, the lymphatics are the usual channel of communication between the place of infection-the sore-and the system. The spirochate has been found in the glands within a few hours of infection, and before the appearance of any sore. Neisser and others have, in experiments on apes, found the spirochaete in the neighbouring glands before the appearance of any induration in the chancre; and they have infected other apes with syphilis from the products of these glands. What effect, therefore, can excision or the cautery have on the prevention of syphilis, which probably occurs concurrently with the earliest formation of the chancre? Such treatment causes pain and discomfort, and tends to confuse rather than help the issue. It is true that in the Freneh and German armies the number of cases of primary syphilis have been much reduced since it became the custom after exposure to compel the soldier to lubricate the parts concerned with a 20 per cent. calomel ointment, and to take other disinfecting precautions; but to be of any avail this must be carried out within 'two or three hours of contact.

The best local treatment is the application of a finelypowdered preparation of one of the odourless forms of iodoform with boric acid with which a 15 per cent. strength of calomel is intimately mixed; sometimes the old-fashioned black wash answers better.
Sloughing Syphilitic Phagedaena.

This condition, fortunately of rare occurrence in early syphilis, is sometimes very alarming, and demands prompt treatment. It occurs in the robust quite as often as in the cachectic and weakly subject. It is oftenest seen in cases of chancre concealed under a phimotic prepuce, and, in my experience, in association with an accompanying gonorrhoea. These two maladies not infrequently occur together, and I have on several occasions witnessed the development of a hard sore whilst treating a patient for gonorrhoea. Ulceration of a primary sore is not uncommon, but rapid spreading of the ulceration is a significant sign, and it is often followed by a destructive gangrenous inflammation of surrounding tissues. I have seen a sore, apparently quite benign, becone $\theta$.within a week a spreading sloughing inflammation, threatening not only the surrounding skin but destruction of the penis itself. These cases are usually treated with strong caustic and escharotic applications, which, however, cause much anxiety, pain, and depression. I would emphasize the value of treatment by immersion in hot baths, associated, of course, with the internal administration of mercury. The affected part may be immersed in a vessel in bed, although in severe cases I much prefer the use of the hot. sitz bath in which some boric acid or other mild disin. fectant is dissolved. I have kept patients as long as three weeks in such a bath, although, if desired, a few hours' sleep in bed may be allowed. It is surprising how gratifying the result of this treatment is. Instant comfort. is experienced, pain disappears, little inconvenience is felt, and within a short time an improvement in the local condition is seen. A reliable nurse to maintain the supply of hot water and to keep the patient in blankets is essential. I have never seen a case fail to respond to this treatment. Two cases will illustrate it.

Sloughing Syphilitic Phagedaena with Gonorrhoea.

CASE I.-A man, aged 38; was seen in consultation with Dr. H. on October 7th, 1905. He was supposed to be suffering from gonorrhoea and had been under treatment seven weeks. The prepuce had been slit up for phimosis. The temperature had suddenly risen and his penis had become swollen and inflamed. $I$ found him very ill. He was in great mental and physical I found him very ill. He was in great mental and physical penis presented an alarming appearance : extensive sloughs penis presented an alarming appearance: extensive sloughs involved both the skin, prepuce, glans, and scrotum; there was a hard mass of ulceration, difficult to define, on the right of the
glans, evidently a primary sore. Gonococci were afterwards glans, evidently a primary sore. Gonococci were afterwards Masses of painful enlarged glands occupied both groins, and the glands in the axillae and neck were hard and swollen. $\mathrm{He}$ had a large painful periosteal swelling on the forehead, sym. metrical grey ulceration on his tonsils, and a few scattered papular spots on his body. Potassium iodide and mercury were. prescribed in small doses, and he was placed in a hot sitz bath. in which he remained for three weeks. Relief was almost immediate; there was no further spreading, the slough awa losing a away leaving a healthy granulating surface, and the disfigure-
ment was less than anticipated. In five weeks he was able to. walk out of doors.

CASE II.-A man, aged 22, was treated in the Hulme Military Hospital, where he had been an in-patient for six weeks before. coming under my care in December, 1900 . He had had gonorrhoea, but during convalescence an induration had appeared the prepuce, causing phimosis, for which circumcision had been performed. When seen by me his penis wos engorged, and spresding, slonghing ulceration involved the spreading, sloughing ulceration involved the glans and a considerable portion of the penis. The inguinal glands were enlarged, the throat was red and inflamed, and he had a
roseolous eruption on the body. Immediate relief was afforded by immersion in a hot sitz bath in which he remained night and day for eighteen days, in this way and with intramuscular injections of grey oil, the symptoms were quickly overcome.

The Occurrence of Syphilis without a Local Sore.

Cases of secondary syphilis occasionally occur in which no trace of a primary sore can be found. How this comes: about-how the blood becomes directly. infected-it is difficult to explain. I have seen several such cases.

Syphilis without Discoverable Chancre.

A man, aged 30, consulted me in January, 1906, for loss of hair and small scaly papules on his scalp. He had no idea of syphilis, and contemplated marriage in three months. Similar spots were found on his body, ulcers in the mouth and on the tonsils, and enlarged painless glands in both groins and in the occipital and cervical regions. The possibility of infection was frankly admitted, but no sign of chancre could be discovered in any part of his body nor in the urethra. The symptoms rapidly declined under a mercurial course. 
Secondary Syphilis without Obvious Chancre.

I was consulted on February 12th, 1900, by a man, aged 34 who had mucous patches in the mouth, ulceration on the tonsils, enlarged glands, and a roseolous eruption on the chest. No sign of a primary sore could be found, but he responded readily to mercurial treatment.

\section{Extragenital Chancre.}

Cases of extragenital chancres, too, are often very confusing. That they occur on the lip, the eyelid, nipple, finger, tonsil-in fact, wherever there is skin or mucous membrane-is well known; but it is none the less true that when confronted with such cases we are apt to over. look their nature. Several striking illustrations could be given. It is, however, to the "hidden" chancre that I wish to draw attention. Syphilis may be suspected, search made, and yet the chancre remain undiscovered unless great care is taken. It is well in obscure cases to strip the patient entirely. Two cases have recently come under my care:

\section{Cases of "Hidden" Chancre.}

CASE I.-A man aged 40 was sent to me by Dr. H. for a troublesome eczema of the scrotum. He was married, and had been well until six weeks before. The scrotum was red, inflamed, and scaly. Small ulcers were noticed on the tongue and tonsils, enlarged glands in the neck and groins, and on stripping faint roseolous eruption on his body. No sore could at first be found on the penis or elsewhere. On making him bend, however, to see if any condylomats existed, a small indurated, slightly eroded sore was seen on the left side at the junction of the scrotum with the perineum. He is rapidly recovering under intramuscular injections of grey oil.

CASE II.-Another patient, aged 27, saw me on January 12th, 1910, with undoubted signs of secondary syphilis. He admitted exposure, but no local sore could be found. He wore a "body bolt," which carelessly I omitted at first to have removed. When this was done a well-marked indurated sore was noticed in the mid-abdominal line 1 in. below the ambilicus.

\section{Difficulties in Treating Malignant Syphilis.}

By this term is meant cases of exceptional severity, in which secondary symptoms develop, progress, and succeed one another with alarming rapidity, produce profound illness, and resist ordinary treatment. It is probable that the severe nature of the symptoms is due to an idiosyncrasy in the patient-a peculiar constitutional susceptibility to the poison-rather than to any difference in the virus itself. It is apparently not due to the state of health or to the age of the patient, as it occurs, in my experience, as often in the strong and robust as in the weak and ill. developed-and in the young and old as often as in those of middle life. In some cases it is influenced perhaps by insufficient treatment; but it occurs also in spite of sound treatment vigorously applied. It does not necessarily follow a severe local sore. Some of the most intractable and extensive sores that I have seen have been followed by mild syphilis. The following cases are instructive in this connexion.

\section{Malignant Syphilis Resembling Small-pox}

A man, aged 29, was seen in bed on January 11th, 1897. He had been ill a week with feverishness and headache, and two days before he noticed "spots" on his body. The temperature was $104^{\circ}$ : pulse 120 . He had intense headache, backache, and waing in the legs. On the face. body, and extremities was a propains in the legs. On the face. body, and extremities was a prosome vesicular, others pustular. Many were of "shotty " hardsome vesicular, others pustular. Many were of " shotty" hardness, and showed umbilication. The tongue was furred and dry, pox. Quite accidentally enlarged glands were noticed in his groins, and inspection of the penis revealed an indurated, ulcerating sore on the right side of the prepuce. At first he gtrenuously denied exposure, but later admitted an indiscretion two months before his illness began. So strong was the resemblance to small-pox that, notwithstanding the evidence of gyphilis, I asked Dr. Niven to see him, who agreed that we were justified in dismissing the suspicion. He was at once given relieved. His spots, however, crusted over, becoming rupial; and, notwithstanding the mercurial treatment, he developed and, notwithstanding the mercurial treatment, he developed nodes on the forehead and occiput. It was not until potassium nodes on the forehead and occiput. It was not until potassium on the specific symptoms. He remained under continuous treatment for a year, and then foolishly disappeared. In March, 1899, he returned in a shocking state. He was cachectic; there was phagedaenic ulceration of the throat; a considerable portion of the roof of the mouth was ulcerating and bone was laid bare; he had a large gummatous swelling on his hesd and gummatous ulceration on the right leg. 'He took potassium iodide and mercury for many months, also arsenic and quinine, before his symptoms subsided and he was able to resume his work. Since then I have lost sight of him. One regrets, that this case came under observation before I was familiar with the intramuscular treatment of syphilis. The familiar with the intramuscular treatment of syphilis. The case also illustrates the early period at which periosteal happens much more frequently than is generally imaginedhappens much more frequently than is generally
and also the value of potassium iodide in such cares.

$$
\text { Case of Malignant Rupial Syphilis. }
$$

On May 18th, 1903, I saw a man, H. L., aged 22, who had been under treatment for syphilis for eighteen months previously. During the whole of that time he had been unable to follow his employment. His prescriptions showed that he had taken at different times grey powder, potassium iodide, Fowler's and different times grey powder, potassium iodide, Fowler's and Donovan's solutions, and other remedies, but he told me that whatever he took disagreed with him, causing either vomiting, diarrhoea, or coryza, and that he had never been able to con-
tinue treatment for more than a few weeks at a time. His tinue treatment for more than a few weeks at a time. His
nose, upper lip, and a portion of the right cheek were destroyed nose, upper lip, and a portion of the right cheek were destroyed
by an ulcerating lupoid-looking mass; half his penis had gone, his body and extremities were covered with rupial sores, his right eye showed extenfive iritic adhesions. Under a course of intramuscular injections of grey oil as used by Lambkin, which were well borne, he st once began to improve, and in eighteen months he had resumed work, though of course years, and when last heard of had had no further relapses.

This case is a good example of the class which seems quite unable to take specific remedies by the mouth, but tolerates them when administered intramuscularly.

The Need to Prohibit "Alcohol" in Treating Syphilis.

It is well to remember the pernicious effect of alcohol when treating cases of syphilis. It appears almost to be a direct antidote to the usual beneficial effect of mercury. So many cases have come under notice in evidence of this, that it is my custom to insist on abstention, except under exceptional circumstances and for limited periods. Spirits and champagne are especially harmful.

Syphilis carefully Treated : Recovery Retarded by Alcohol.

A patient, aged 24, sent to me by Dr. G. on March 6th, 1909 had contracted syphilis two and a half years before. He had taken continuous treatment for the flrst eighteen months and taken continuous treatment for the first eighteen months and intermittent later. He had lost weight and had suffered frequent recurrences. It was elicited that during the whole period of his illness he had taken large quantities of beer and whisky.
On the soft palate above and to the right of the uvula was an ulcerating almost phagedaenic condition with irregular edges, and a large hole communicating with the pharynx. He had enlarged glands in the neck and groins. At the seat of the riginal chancre on the corona was a small hard gumma, and he had a scattered papulo-squamous eruption on his body. As he had albumen in the urine, treatment at first was cautious, but the albumen soon disappeared under treatment. He had four intramuscular injections of 1 grain of calomel, and afterwards weekly and lately fortnightly injections of mercurial cream, which agreed well. He took iodipin tablets and refrained from alcohol. His improvement has been uninterrupted, and from alcohol. His improvem

A case sent to me by Mr. Jonathan Hutchinson was of a similar nature, but the patient would not discontinue alcohol. Several methods of treatment were tried without any good result, and further supervision had to be declined. Other cases, however, that have resisted treatment have begun to improve as soon as alcohol has been stopped.

\section{The Frequency of Affections of the Nervous System in Early Syphilis.}

It is now well known that the cerebro-spinal system is often attacked by syphilis at a much earlier period than was formerly believed. Symptoms due to syphilitic deposits implicating or pressing upon some part of the brain or cord occur during the earlier stages of secondary syphilis. It is well to keep this in mind, and to be prepared to treat such cases vigorously, as they are apt to resist mild treatment; and unless the lesion is rapidly relieved, permanent loss of fanction will be threatened. Vigorous treatment gives the best chance for the return of normal function. How many cases of such a common condition as hemiplegia have their origin in syphilis, and would recover under severe and well-directed treatment! The following two cases illustrate these points:

\section{Right Hemiplegia and Aphasia occurring in Early Secondary} Syphilis.

A man aged 25 came under my care on January 6th, 1906, in consequence of sudden loss of power in the right arm and 
partial aphasia which had occurred during the previous night. The right arm was helpless, and he could not grasp ; there was an area of impaired sensation on the back of his right forearm and hand, and his speech was affected. There was no albumen in the urine, and the heart was sound. It was ascertained that he had contracted syphilis six months previously, and that he had gone through a course of injections in Paris, a second course being shortly due. Six injections of gr.j calomel were given at intervals of four days intramuscularly and afterwards each at intervals of four days intramuscularly, and afterwards each Recovery was complete and speedy; in three months all sym. pecovery has disappeared, nor have they returned. Treatment ptoms had disappeared, nor
was continued for two years.

Diabetes Insipidus and other Nerve Phenomena due to Early Syphilis Cured by Intensive Treatment.

A man aged 29 consulted me on September 23rd, 1885, complaining of great thirst, frequent and copious micturition, severe headache, occasional vomiting, weakness, and defective vision of the left eye. He had been married seven years, and had two healthy children. He denied syphilis, but admitted gonorrhoea two years before, which was very obstinate to cure, and for which he took much medicine. His present illness began six months ago, with occasional headaches, which now were months ago, with occasional headaches, which now were frequent and severe. Latterly he had passed large quantities of urine, rising hourly in the night; he had inordinate thirst, and had lost weight. When seen he was in bed-dull, apathetic, anaemic, sick, and suffering intense vertical headache. The rregular, tongue dry and brown. He was passing between 8 and 10 pints daily of pale, straw-coloured, clear, odourless urine, speciffc gravity 1003, which contained neither albumen nor sugar. The left disc showed well marked optic neuritis; the right appeared normal. The deep reflexes were exaggerated. The symptoms were regarded as due to gross intracranial syphilitic disease; probably a multiple lesion. The polyuria was looked on as a secondary phenomenon most likely cansed by some gummatous deposit pressing upon the floor of the fourth ventricle and implicating the controlling centre. He was treated with gradually increasing doses of potassium He Was treated with gradually increasing doses of potassium part of October a large periosteal swelling developed on the top of his head, thus confirming the specific nature of his illness. Mercurial inunctions were substituted for the perchloride, and the iodide was rapidly increased until he was taking drachm doses three times daily. He became ptyalized early in November, and from that date his recovery began. The headaches subsided, the polyuria and the node gradually disappeared, the optic neuritis cleared up, and by the end of January he was able to resume work. He subsequently took small doses of mercury for two years, and he was quite well fifteen years later when he left Manchester.

This case not only demonstrates the need for the inten. sive treatment of syphilis when it attacks the nervous system, but is an example of the value of those cardinal rules of Dr. Hughlings Jackson (the greatest of all neurologists), who used so often to say:

If you see a mixture of nerve symptoms which do not appear to coincide with each other-a medley of symptoms, an incoherent succession or a random association of symptoms apart from any demonstration of actual syphilis-suspect it and treat it.

Treatment of Early Syphilis.

A study of the fow cases that have been related under the previous headings show that the treatment has varied. Each case of syphilis should be treated on its own merits, and if one method causes no ready response, some other line must be adopted. Age, sex, constitution, resisting power, the condition of the various organs, the nature and severity of symptoms ; the means, leisure, and the surroundings of the patient; the effect of treatment on the mind, constitution, and on the course of the disesse-all these points must be considered in the effort to cure our patient. There is no routine way of dealing with every case.

Mercury remains our sheet anchor. Its continuous administration in small doses over a long period-never less than two years-has in ordinary cases given me the best results. To treat only when symptoms appear is to court disaster - the enemy is at the gates, and may renew his attack with overwbelming strength; why not con tinue to harass, wound, or destroy him after his first repulse? Neither has it ever been clear to me that the intermittent administration of the drag increases its potency and value, nor that its continuous employment reduces it to impotence; a patient at the end of a long and continuous treatment is as liable to become ptyalized if his limit is exceeded, as he was at the beginning of his course.

Fortunate in early days in being a pupil of Sir Jonathan Hatchinson, my treatment until the last ten years was entirely on his lines-namely, small doses of grey powder by the mouth given over a long period, supplemented by opium, iron, quinine, arsenic, and potassium iodide, as each individual case required. I never treat for less than two years; often much longer. As a whole the results bave been excellent. Reflection, however, brings to mind a few cases, treated years ago, which undoubtedly would bave done better had I had the advantage of the knowledge and experience at our disposal now. The intenser effect of the intramuscular treatment in intractable cases bas greatly impressed me. A notable illustration of its special advantage is seen in the case of $H$. L., described in the notes to malignant syphilis. I never hesitate to employ this method now, directly a resistance to oral methods is manifest. It is usually quite safe to give four injections of 1 grain of calomel in four weeks and 1 grain of metallic mercury fortnightly atterwards for a year, and monthly later as long as is necessary. Of course, circumstances may require some modification of this. The urine, weight, gums, and blood require carefal watching; nodules must absorb before a second injection is given into the same buttock, and the most scrupulous antiseptic technique must be carried out, otherwise disaster will follow. In over 2,000 injections only 2 cases of ill effects have occurred to me-both severe ptyalism. They occurred in hospital in earlier experience, when injections were repeated oftener, and before the extreme watchfulness necessary had been fully appreciated. No case has caused me anxiety since. The formulae of the creams that I u\&e are those of Lambkin-the creo-camphor in which prevents or greatly relieves the pain; the aseptules supplied by Messrs. Oppenheimer are handy and reliable. This method of administration is desirable in other than refractory cases. It is largely employed in the army, and in private practice it is useful when the existence of the disease must be kept secret; also in dyspeptic subjects, and when mercury given by the moutb, no matter in what combination, causes colic or diarrhoea.

\section{Treatment by Arylarsonates Alone and in Combination with Mercury.}

Much has been published lately regarding the ex. ceptional value of arylarsonates or their combination with mercury in the treatment of syphilis. In my own practice these drugs have been held back until lately, partly because from time to time, serious, even calamitons, results bave been published as a consequence of their exhibition; also because, although their action on symptoms has in many recorded cases been phenomenal, a period of years must elapse before their lasting curative property is estab. lished; and, lastly, because in difficult cases I have bc en satisfied with the injections of calomel or grey oil alone, or associated with the internal administration of potassium iodide, arsenic and quinine.

No doubt you all saw a letter from Mr. J. Ernest Lane in the British Medical Journal (March 5th, 1910) in which he describes a case of syphilis in his own practice, in which at the end of a course of ten injections of orsudan, given in 10 grain doses on alternate days, the patient suddenly became blind from optic atrophy, and in the same letter 3 cases are mentioned as experiencing a similar fate after soamin. Again, in the same JoUnNaL of January 22nd, 1910. Drs. Lundie and Blaikie relate a corresponding tragedy after a course of soamin; and in the Lancet, 1909, p. 1196, Dr. Bagshawe sounds a warning note regarding the danger of atoxyl.

For a detailed account of a remarkable series of cases of amblyopia and blindness following the use of atoxyl and arsacetin, reference should be made to the Bulletin of the Sleeping Sickness Bureau (No. 8, 1909). In this Professor Dr. Max Beck notes that Watermann treated four cases of tabetic optic atrophy with atoxyl, and in each case the vision became markedly worse. He also describes 23 cases of complete and 7 cases of partial blindness out of 1,633 patients treated with atoxyl at Sesse by the members of Koch's expedition. Drs. A. Van Gehuchten and A. Tils describe the case of a man (infected by trypanosomes) who became blind after treatment with atoxyl. Two series of cases treated by Gray with atoxyl are also recorded. In the first 1,135 cases $47 \mathrm{had}$ alterations of vision and 20 became totally blind. In the second series of 1,712 cases 70 had dimness of vision and 10 became totally blind; in 2 of the latter mercury was given with atoxyl. Dr. Paderstein ${ }^{1}$ has published 12 cases treated with atoxyl 
and 2 with arsacetin, all followed by amblyopia varying in severity from actual blindness to marked contraction of the field of vision. Paderstein's patients were Europeans. Those of Beck and Gray were natives of Uganda.

On the other hand many others have. employed the arylarsonate salts without any ill effects and with conspicuous advantage. In the same article from which the above is quoted, Major Ward is reported to have given 18,000 grains of soamin to more than 100 syphilitic patients during the last two years without any ill effects. Colonel Lambkin, so zealous a worker in this field, has treated 308 cases with arylarsonates without any bad effect. Daring 1909 he gave over 2,000 intramuscular injections of soamin and arsacetin with no ill consequence and he attributes the toxic symptoms that have been described to the liability to decomposition that occurs when these salts are kept in solution. Beck, however, regards the eye symptoms as due to chronic poisoning dependent on idiosyncrasy, as many patients receiving larger quantities of similarly prepared arylarsonates escape eye symptoms.

Colonel Lambkin is the pioneer in treating early syphilis intramuscularly with the arylarsonates in combination with mercury. $\mathrm{He}$ related his earlier results at the Belfast Meeting of the British Medical Association, 1909." Since then he has recorded an interesting series of case treated with a preparation of atoxyl and mercury made for him by Messrs. Greef of Charlottenburg, Germany and named mercury atoxylate. $\mathrm{He}$ has already treated a large number of cases with remarkable success and without the slightest toxic effect. He gives about 9 grains in eight injections, with three to seven days' interval between each, and repeats the course after one month's rest. ${ }^{8}$. Through the courtesy of Colonel Lambkin I was privileged last December to see the technique and effect of this treatment at the Rochester Row Military Hospital His results were very striking.

Only in exceptional cases have I felt justified at this stage in employing the remedy, but I have done so in 5 cases, in 4 of them with marked benefit. The fifth case is one of general paralysis, and this, too, shows improvement.

The first is one already alluded to (H. G.) in speaking of relapsing chancre. This recurred notwithstanding intramuscular treatment with grey oil, but the chancre disappeared under one course of injections of atoxylate of mercury.

The second case, also already mentioned, was a man (A. B.) with a coppery squamo-papular eruption all over his body, throat implications, and enlarged glands; about to marry. $\mathrm{He}$ is improving most strikingly under a course of mercury atoxylate.

The third case is that of a gentleman under treatment by intramuscular injections of grey oil for eight months, who developed a very extensive papulo-squamous eruption on the back, which neither potassium iodide nor Fowler's solution given at the same time as the injections appeared to benefit. It cleared up entirely during one course of mercury atoxylate.

The fourth case is one of very severe secondary syphilis, in which the throat was sloughing, an angry spreading sore existed, and the patient was cachectic and ill. He has improved rapidly under a course of the mercury atoxylate.

The mercury atoxylate* has a wonderful immediate effect on intractable symptoms, and so far I have noticed no ill effects. Further experience will show if it confers equal fature benefits. These new remedies should not be abandoned because of untoward accidents alone. The early employment of mercury caused many calamities, but it has outlived opposition. We should rather redouble our efforts and caution and strive to appreciate the class of case in which these new drugs can be administered with additional benefit, and how they can be given without harm.

My experience with the intramuscular injection of the soluble salts of mercury is limited to their administration to patients temporarily resident in Manchester, but under the care of foreign specialists. They appear to cause more pain ",nd depression, and to be less effective in the removal of symptoms, than the methods already described.

Mercurial inunctions alone and in combination with notassium iodide have often served me well, especially in "melting away" those large abdominal masses (called

* Messrs. Oppenheimer and Son have procured the mercury atoxylate from the German firm and put it up for me in aseptules ready for use with Lambkin's cream basis. They assure me that if the aseptules are not tampered with, and if they are kept in a dark place, there is no tuberculous, but which I regard as oftener due to hereditary syphilis) so frequently seen in children. It is, however, a dirty and, if skilfully carried out, an expensive performance.

Potassium iodide is a drug we could not do without. Numbers of cases of secondary syphilis respond to its influence which resist mercurial treatment when given alone. Its value in the absorption of large specific masses is incontestable.

In 1898 I saw a young man, aged 22, who had been in the Royal Infirmary under the late Dr. Thomas Harris and discharged as incurable with a growth in the right chest. He was breathing almost entirely with his left lung-the right percussion note in front and behind being absolutely dull-with almost inaudible breath sounds, and no fluid. His right arm was inaudible breath sounds, and no fluid. His right arm was rapidly increasing doses of potassium iodide and mercury, the whole malady disappeared. He had not had syphil
himself, but there were reasons to assume hereditary syphilis.

I showed this man subsequently to Dr. Harris, and he was much impressed. Many other successful cases could be cited, especially one of large liver gummata, and another lung mass, which responded to treatment by iodide. In some cases the potassium or sodium iodide agrees badly; in these iodipin, an iodized oil, is a valuable substitute. It is prepared in two strengths-10 per cent. and 25 per cent. It can be given intramuscularly-or by the mouth in the form of capsules, tablets, or in milk. It is well tolerated, and my results justify the reputation that it has earned in other hands.

$$
\text { REFERENCES. }
$$

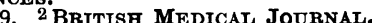
1909, vol. ii, p. 380 . B Ibid., January 1st, 1910.

\section{THE SURGICAL TREATMENT OF GASTROPTOSIS.}

Being an analysis of a Series of Twenty Cases, wi Special Reference to the Results of Operative Treatment.*

By FREDERIC EVE, F.R.C.S., SURGEON TO THE LONDON HOSPITAL.

THE symptoms sometimes associated with gastroptosis are those common to several other affections of the stomach. Hence the diagnosis rests on physical examination and on a process of exclusion, the culminating point of which is in some cases an exploratory laparotomy.

The patient suffers from severe chronic dyspepsia, often of long standing. In two of my cases the symptoms have lasted twenty years, in one for twelve, and in two for ten. There is flatulent distension, a sense of fullness, and pain after food referred to the epigastrium and sometimes radiating under the right costal margin and to the scapula. The pain comes on at various periods after food, usually early, but occasionally after a longer period, even up to three or four hours. Vomiting is often present, and, in exceptional cases, is incessant. The symptoms may be more or less persistent, but commonly the sufferer is subject to periodic attacks or exacerbations of the symptoms which lead to their admission to hospital on several separate occasions. The pain is prone to be made worse by standing or walking about after a meal. During the exacerbations there is in some cases superficial and deep tenderness over the epigastrium. The diet is usually, by force of circumstances, restricted. Meat is often avoided and the worst cases restrict themselves to milk, eggs, and such light diet in small quantities. Haema. temesis is present frequently; it existed in nine, and perhaps ten, out of my twenty cases. In seven of these it was considerable in amount-a teacupful or more; exceptionally, it was frequent and small in quantity. The following cases are good examples :

Mary C., aged 46, suffered from pain, usually four hours after food, which was relieved by vomiting. She had repeated attacks of haematemesis "in large amounts" twenty years, seven years, and one year and nine months before admission respectively.

Emily S., aged 23, dairymaid. In 1907 she began to suffer after food with attacks of pain, which passed through to the back, also with nausea and flatulence. Six months later she

* Read before the Medical Society of London. 